\title{
Nutritional composition of the diets of South Asian, black African-Caribbean and white European children in the United Kingdom: The Child Heart and Health Study in England (CHASE)
}

\author{
A. S. Donin ${ }^{1}$, C. M. Nightingale ${ }^{1}$, C. G. Owen ${ }^{1}$, A. R. Rudnicka ${ }^{1}$, M. C. McNamara ${ }^{1,2}$, C. J. Prynne ${ }^{3}$, \\ A. M. Stephen ${ }^{3}$, D. G. Cook $^{1}$ and P. H. Whincup ${ }^{1}$ \\ ${ }^{1}$ Division of Community Health Sciences, St George's, University of London, London SW17 ORE, UK \\ ${ }^{2}$ Food Standards Agency, London WC2B 6NH, UK \\ ${ }^{3}$ Medical Research Council Human Nutrition Research, Cambridge CB1 9NL, UK
}

(Received 8 July 2009 - Revised 6 January 2010 - Accepted 22 January 2010 - First published online 16 March 2010)

In the UK, South Asian adults have increased risks of CHD, type 2 diabetes and central obesity. Black African-Caribbeans, in contrast, have increased risks of type 2 diabetes and general obesity but lower CHD risk. There is growing evidence that these risk differences emerge in early life and that nutritional factors may be important. We have therefore examined the variations in nutritional composition of the diets of South Asian, black African-Caribbean and white European children, using $24 \mathrm{~h}$ recalls of dietary intake collected during a cross-sectional survey of cardiovascular health in eighty-five primary schools in London, Birmingham and Leicester. In all, 2209 children aged 9-10 years took part, including 558 of South Asian, 560 of black African-Caribbean and 543 of white European ethnicity. Compared with white Europeans, South Asian children reported higher mean total energy intake; their intakes of total fat, polyunsaturated fat and protein (both absolute and as proportions of total energy intake) were higher and their intakes of carbohydrate as a proportion of energy (particularly sugars), vitamin $\mathrm{C}$ and $\mathrm{D}, \mathrm{Ca}$ and haem $\mathrm{Fe}$ were lower. These differences were especially marked for Bangladeshi children. Black African-Caribbean children had lower intakes of total and saturated fat (both absolute and as proportions of energy intake), NSP, vitamin D and Ca. The lower total and saturated fat intakes were particularly marked among black African children. Appreciable ethnic differences exist in the nutritional composition of children's diets, which may contribute to future differences in chronic disease risk.

Ethnicity: South Asian children: African-Caribbean children: Diet: 24-Hour recalls

Compared with white Europeans, South Asians living in the UK have increased risks of CHD, type 2 diabetes and central obesity $^{(1-5)}$. Black African-Caribbeans living in the UK have increased risks of type 2 diabetes and general obesity but have lower risks of $\mathrm{CHD}^{(2,3,5,6)}$. Ethnic differences in the nutritional composition of diets could play an important role in these differences in disease risk, which are currently unexplained ${ }^{(7)}$. Nutrient composition is important in the aetiology of CHD, type 2 diabetes and obesity ${ }^{(8)}$. In particular, excessive consumption of energy-dense foods is associated with increased risks of overweight and obesity ${ }^{(8)}$, while high total and saturated fat intakes increase risks of insulin resistance ${ }^{(9)}$, impaired glucose tolerance ${ }^{(10)}$, raised LDL-cholesterol levels and $\mathrm{CHD}^{(11)}$. High intakes of sugars may increase serum TAG and reduce HDL-cholesterol ${ }^{(12-14)}$. Low fibre (NSP) intake is associated with higher insulin resistance, raised total and LDL-cholesterol and an increased risk of obesity ${ }^{(15,16)}$ and stroke $^{(17)}$. Low intakes of micronutrients, including folate ${ }^{(8)}$, vitamin $\mathrm{C}^{(18)}$ and vitamin $\mathrm{D}^{(19)}$, have been associated with increased risks of CVD and type 2 diabetes. The nutritional composition of adult diets differs between ethnic groups in the UK, in ways that could contribute to explaining ethnic differences in disease. In particular, intakes of total and polyunsaturated fat have been reported to be high among South Asians ${ }^{(20-22)}$; a recent report has suggested that saturated fat intakes may now also be higher ${ }^{(23)}$. Intakes of fruit and green vegetables may be low in South Asians ${ }^{(24)}$, though this pattern has not been observed in all studies ${ }^{(3)}$. In contrast, among black African-Caribbeans, intakes of fat (particularly saturated fat) may be low and carbohydrate intake high ${ }^{(3,20,22,25)}$.

There is growing evidence that the differences in chronic disease risk between South Asians, black African-Caribbeans and white Europeans may originate early in life. Compared with white Europeans, British South Asian children and adolescents have markedly increased risks of clinical type 2 diabetes $^{(26)}$ and have higher fasting insulin levels from about 10 years of age onwards ${ }^{(27,28)}$. Higher prevalences of overweight and obesity have been reported in South Asian and black African-Caribbeans in childhood and adolescence ${ }^{(3,29,30)}$. However, little is known about the nutritional composition 
of diets among British children of different ethnic groups. We therefore carried out a detailed assessment of nutritional composition during a recent survey of cardiovascular health in 9- to 10-year-old children of different ethnic origins. We report here on ethnic variations in nutritional composition between South Asians, black African-Caribbeans and white European children, and also present data separately for South Asian children of Indian, Pakistani and Bangladeshi origin, and for black African-Caribbean children of African and Caribbean origin.

\section{Subjects and methods}

The Child Heart Health Study in England (CHASE) is examining the cardiovascular health of 5000 9- to 10-year-old children of white European, South Asian and black AfricanCaribbean origin living in England. The investigation of diet and nutrition was carried out in a sub-study of approximately 2000 children (CHASE-2000) studied between February 2006 and February 2007. Ethical approval was obtained from the relevant Multi-Centre Research Ethics Committee. Participating schools were identified on the basis of the pupil ethnicity, using information obtained from the UK Government Department for Education and Skills. All state primary schools in London, Birmingham and Leicester with between 15 and $50 \%$ pupils of white European origin were identified. Two separate random samples of forty-three and forty-two schools were taken including (a) schools with a high prevalence $(20-80 \%)$ of South Asian pupils, stratified by Indian, Pakistani and Bangladeshi origin, and (b) schools with a high prevalence $(20-80 \%)$ of African-Caribbean pupils, stratified by African and Caribbean origin. Schools that did not agree to participate $(30 \%)$ were replaced by a school with a similar ethnic composition and in the same borough. Invitation letters (translated where necessary) were sent to parents or guardians of pupils in year 5 classes.

A single research field team visited schools in different areas in rotation during school terms in order to carry out survey assessments. Parents completed a questionnaire on their self-defined ethnic origin and that of the child and on occupation and educational attainment. Children provided information on parental and grand-parental place of birth. Ethnicity was defined on the basis of parental self-defined ethnicity for both parents, or (where not available) parentally defined child ethnicity, or (where not available) child-defined geographic origin of parents and grandparents. In the present analyses, 'white European' includes children whose ethnic origin was defined as 'white British', 'white Irish' and 'white European' (or a combination of these) and excludes 'white other'. 'South Asian' includes 'Indian', 'Pakistani', 'Bangladeshi' and 'Sri Lankan' (or a combination of these). 'Black African-Caribbean' includes 'black African', 'black Caribbean', 'black British' and 'black other' (or a combination of these). The 'other' ethnic group includes all other categories of individual and mixed ethnic origins.

Weight and height were measured by one of three research nurses, weight with an electronic digital scale (Tanita Inc., Tokyo, Japan) and height (measured to the last complete millimetre) with a portable stadiometer (Chasmors Ltd, London, UK); BMI (weight $(\mathrm{kg}) /$ height $\left(\mathrm{m}^{2}\right)$ ) was calculated. Food intake was assessed by two research nutritionists
(A. S. D. and M. C. M.). The $24 \mathrm{~h}$ recall method was chosen because it provides valid information in children in this age group ${ }^{(31-34)}$, is suitable for large-scale survey use $^{(35)}$, and does not require high levels of literacy. A single, structured $24 \mathrm{~h}$ dietary recall interview was carried out, which followed the recommendations of the Nordic Cooperation Group of Dietary Researchers ${ }^{(36)}$ and included several elements of the United States Department of Agriculture (USDA) multiple-pass method ${ }^{(37)}$. All interviews were carried out in English (the language in which all children were being taught) in private, without a carer or teacher present. The interviewer orientated the child on the previous day, enquiring about events and activities in which the child had participated $^{(32)}$. A list of foods and beverages consumed the previous day was constructed, together with the time and occasion on which each item was consumed. A detailed description was made of the food consumed. Photographs of food items and different portion sizes were used ${ }^{(38)}$ to help the child report the quantity of food consumed. Final probing was carried out for any forgotten items, particularly drinks and snacks. Questions were asked to identify whether this was a 'usual' day in terms of dietary intake for the child. The data were then entered and analysed for nutrient composition by experienced dietary coders at the Medical Research Council Human Nutrition Research Centre (MRC-HNR) using the Diet In Nutrients Out (DINO) package, which generates a food code and an associated weight for each item of food and drink recorded. Age-specific portion sizes were determined from the National Diet and Nutrition Survey of Young People ${ }^{(27)}$. Food and nutrient intakes were calculated using the in-house food composition database based on McCance and Widdowson's The Composition of Foods, 6th edition $^{(39)}$. The nutrient contents of foods that were not already in the DINO database were calculated from recipes obtained from various sources including the second supplement to McCance and Widdowson's The Composition of Foods 1985: Immigrant Foods ${ }^{(40)}$, the MRC-HNR in-house database of food composition tables for the Gambia and authoritative international recipe books ${ }^{(41,42)}$.

In order to identify potential under- and over-reporters, standard equations were used to estimate BMR which are age and sex specific and based on the measured weight of the child ${ }^{(43)}$. These were multiplied by lower and upper cut-off physical activity levels (PAL) of $1 \cdot 1$ and 2.2 (assuming an average PAL of 1.55$)^{(44)}$ to provide estimates of minimum total energy expenditure (to identify probable under-reporters) and maximum energy expenditure (to identify probable overreporters). These estimates were compared with reported energy intakes, and, if found to be below the estimated minimum expenditure or over the maximum expenditure, children were identified as under-reporters or over-reporters, respectively ${ }^{(45)}$. Participants identified as under-reporters or over-reporters were included in the main analysis but were excluded in specific sensitivity analyses (reported in the Results section).

\section{Statistical analysis}

All analyses were carried out using STATA (version 10.1; StataCorp LP, College Station, TX, USA). Distributions of nutrient composition variables were examined for normality and 
log transformed where necessary. Multilevel linear regression models taking account of the natural clustering within school were used to provide adjusted means and ethnic differences in nutrient composition (using XTMIXED and LINCOM commands). All analyses adjusted for sex, age in quartiles, month, day of the week and interviewer, all as fixed effects.

\section{Results}

Overall, 2255 children (mean age 9.9 years, 95\% reference range 9.2, 10.7 years) participated, with an overall response rate of $70 \%$. Response rates were slightly higher among white Europeans (71\%), South Asians (73\%) and other ethnic groups (70\%) compared with black African-Caribbeans $(65 \%)$. Among the 2255 participants, 2209 (98\%) provided information of acceptable quality ( 1065 boys and 1144 girls; 558 were South Asian, 560 black African-Caribbean, 543 white European, and 548 of other ethnic origin). Recall assessments could not be completed in forty-six children because the child did not cooperate (n 37), had language difficulties $(n 5)$ or because of time constraints $(n 4)$. The prevalence of under-reporting was $21 \%$ (24\% of boys, $19 \%$ of girls) and over-reporting was $6.7 \%$ (4.7\% of boys, $8.7 \%$ of girls). Under-reporting prevalence varied by ethnic group; $27 \%$ of black African-Caribbean children, $21 \%$ of white European children, $15 \%$ of South Asian children and $23 \%$ of children of other ethnicity were under-reporters. As expected, underreporters were heavier than other children (mean difference
$6.7(95 \%$ CI $5.8,7.7) \mathrm{kg}$ ); this difference did not vary markedly between boys and girls or between children of different ethnic groups.

The nutritional composition of the whole study population is summarised in Table 1. Mean total energy intake was higher in boys, though the proportions of energy derived from fat, carbohydrate and protein were similar in both sexes. Among micronutrients, intakes of vitamin $\mathrm{B}_{12}$ and $\mathrm{Fe}$ were higher among boys. Nutrient composition did not vary appreciably with age over the narrow age range studied (data not presented), but varied slightly by interviewer. Nutritional composition varied by month of the year, but there was no consistent seasonal trend (data not presented). Nutritional composition also varied by day of the week; total energy intakes and proportions of energy derived from fat and protein were higher on Sundays than on weekdays while micronutrient intakes tended to be lower (data not presented). Adjustments were made for month, day of the week and interviewer in subsequent analyses.

\section{Nutritional composition of diets among children from different ethnic groups}

Data on the nutritional composition of the diets of children of South Asian, black African-Caribbean, white European and other ethnic origin are presented in Table 2, in analyses which take account of differences in age and sex, month of year and day of week, with school fitted as a random effect.

Table 1. Nutritional composition of children's diets: by sex

(Mean values and standard deviations or geometric means and interquartile ranges (IQR) ${ }^{\star}$ )

\begin{tabular}{|c|c|c|c|c|c|c|c|c|c|}
\hline & \multicolumn{4}{|c|}{ Boys (n 1065) } & \multicolumn{4}{|c|}{ Girls (n 1144) } & \multirow{2}{*}{$\begin{array}{c}P \\
\text { (difference)† }\end{array}$} \\
\hline & Mean & SD & Geometric mean & IQR & Mean & SD & Geometric mean & IQR & \\
\hline Energy (kJ) & 7856 & 2137 & & & 7646 & 2046 & & & 0.01 \\
\hline Energy (kcal) & 1867 & 509 & & & 1817 & 488 & & & 0.01 \\
\hline Fat $(g)$ & $72 \cdot 3$ & $26 \cdot 9$ & & & 71.0 & $26 \cdot 1$ & & & 0.30 \\
\hline Fat (\% energy) & $34 \cdot 1$ & $6 \cdot 6$ & & & 34.5 & 6.4 & & & 0.11 \\
\hline Saturated fat $(g)$ & $26 \cdot 6$ & $11 \cdot 1$ & & & $26 \cdot 3$ & $11 \cdot 1$ & & & 0.55 \\
\hline Saturated fat (\% energy) & $12 \cdot 6$ & 3.4 & & & $12 \cdot 7$ & 3.5 & & & 0.15 \\
\hline Monounsaturated fat $(\mathrm{g})$ & $24 \cdot 2$ & 9.9 & & & $23 \cdot 3$ & $9 \cdot 3$ & & & 0.03 \\
\hline Monounsaturated fat (\% energy) & $11 \cdot 4$ & 2.9 & & & $11 \cdot 3$ & $2 \cdot 8$ & & & 0.50 \\
\hline Polyunsaturated fat (g) & 13.5 & $8 \cdot 0$ & & & $13 \cdot 7$ & 7.5 & & & 0.61 \\
\hline Polyunsaturated fat (\% energy) & $6 \cdot 4$ & $3 \cdot 1$ & & & $6 \cdot 7$ & $3 \cdot 0$ & & & 0.02 \\
\hline Carbohydrate $(\mathrm{g})$ & $258 \cdot 6$ & $71 \cdot 8$ & & & $251 \cdot 1$ & $68 \cdot 5$ & & & 0.01 \\
\hline Carbohydrate (\% energy) & $52 \cdot 1$ & $7 \cdot 0$ & & & $51 \cdot 9$ & $6 \cdot 8$ & & & 0.49 \\
\hline Sugars $(g)$ & $111 \cdot 5$ & $46 \cdot 0$ & & & $108 \cdot 7$ & $44 \cdot 6$ & & & 0.09 \\
\hline Sugars (\% energy) & $22 \cdot 3$ & $7 \cdot 2$ & & & $22 \cdot 4$ & $7 \cdot 0$ & & & 0.92 \\
\hline Starch, dextrins $(\mathrm{g})$ & $144 \cdot 6$ & $45 \cdot 2$ & & & $140 \cdot 2$ & $42 \cdot 8$ & & & 0.02 \\
\hline Starch, dextrins (\% energy) & $29 \cdot 3$ & $6 \cdot 3$ & & & $29 \cdot 1$ & $6 \cdot 1$ & & & 0.54 \\
\hline Total NSP (g) & 11.7 & 4.9 & & & 11.9 & $4 \cdot 6$ & & & 0.42 \\
\hline Protein $(\mathrm{g})$ & $62 \cdot 3$ & $20 \cdot 6$ & & & $60 \cdot 2$ & $19 \cdot 9$ & & & 0.01 \\
\hline Protein (\% energy) & 13.4 & 3.3 & & & $13 \cdot 3$ & $3 \cdot 3$ & & & 0.39 \\
\hline Vitamin $B_{12}(\mu \mathrm{g})$ & & & 3.0 & $2 \cdot 8$ & & & $2 \cdot 8$ & $2 \cdot 6$ & 0.02 \\
\hline Folate $(\mu \mathrm{g})$ & & & 201 & 117 & & & 198 & 113 & 0.29 \\
\hline Vitamin C (mg) & & & $78 \cdot 6$ & 96.5 & & & 83.8 & $94 \cdot 2$ & 0.09 \\
\hline Vitamin D $(\mu \mathrm{g})$ & & & 1.6 & 1.9 & & & 1.6 & $2 \cdot 0$ & 0.45 \\
\hline $\mathrm{Ca}(\mathrm{mg})$ & & & 718 & 443 & & & 712 & 410 & 0.53 \\
\hline $\mathrm{Fe}(\mathrm{mg})$ & & & $9 \cdot 1$ & $4 \cdot 3$ & & & 8.6 & 3.9 & 0.002 \\
\hline Haem Fe (mg) & & & 0.18 & 0.50 & & & 0.14 & 0.40 & $<0.001$ \\
\hline
\end{tabular}

* Means and standard deviations or IQR are based on the raw data.

$\dagger P$ values for sex difference adjusted for age in quartiles, day of week, month, interviewer and school (random effect). 
Table 2. Nutritional composition of children's diets: by ethnic group*

(Means or geometric means and $95 \%$ confidence intervals)

\begin{tabular}{|c|c|c|c|c|c|c|c|c|}
\hline & \multicolumn{2}{|c|}{$\begin{array}{l}\text { White European } \\
\text { ( } n 543 ; 271 \text { boys) }\end{array}$} & \multicolumn{2}{|c|}{$\begin{array}{c}\text { South Asian } \\
\text { (n 558; } 264 \text { boys) }\end{array}$} & \multicolumn{2}{|c|}{$\begin{array}{l}\text { Black African-Caribbean } \\
\text { ( }(n 560 ; 261 \text { boys) }\end{array}$} & \multicolumn{2}{|c|}{$\begin{array}{c}\text { Other } \\
\text { ( } n \text { 548; } 269 \text { boys) }\end{array}$} \\
\hline & Mean & $95 \% \mathrm{Cl}$ & Mean & $95 \% \mathrm{Cl}$ & Mean & $95 \% \mathrm{Cl}$ & Mean & $95 \% \mathrm{Cl}$ \\
\hline Energy (kJ) & 7634 & 7446,7823 & 8042 & 7842,8242 & 7665 & 7478,7853 & 7688 & 7500,7876 \\
\hline Energy (kcal) & 1814 & 1769,1859 & 1911 & 1864,1959 & 1821 & 1776,1866 & 1827 & 1782,1871 \\
\hline Fat $(\mathrm{g})$ & $70 \cdot 8$ & $68 \cdot 5,73 \cdot 2$ & 76.9 & $74 \cdot 4,79 \cdot 4$ & $69 \cdot 0$ & $66 \cdot 6,71 \cdot 3$ & 70.4 & $68 \cdot 0,72 \cdot 8$ \\
\hline Fat (\% energy) & 34.5 & $34 \cdot 0,35 \cdot 1$ & 35.6 & $35 \cdot 0,36 \cdot 2$ & 33.2 & $32 \cdot 7,33 \cdot 8$ & 34.0 & $33.4,34.5$ \\
\hline Saturated fat (g) & $27 \cdot 2$ & $26 \cdot 2,28 \cdot 2$ & $27 \cdot 3$ & $26 \cdot 2,28 \cdot 4$ & $25 \cdot 0$ & $24 \cdot 0,26 \cdot 0$ & 26.5 & $25 \cdot 5,27 \cdot 5$ \\
\hline Saturated fat (\% energy) & $13 \cdot 2$ & $12 \cdot 9,13.5$ & $12 \cdot 7$ & $12 \cdot 3,13 \cdot 0$ & $12 \cdot 0$ & $11 \cdot 7,12 \cdot 3$ & $12 \cdot 8$ & $12 \cdot 5,13 \cdot 1$ \\
\hline Monounsaturated fat $(\mathrm{g})$ & 23.6 & $22 \cdot 7,24 \cdot 5$ & 25.4 & $24 \cdot 4,26 \cdot 3$ & 23.0 & $22 \cdot 1,23.9$ & 23.5 & $22 \cdot 6,24 \cdot 4$ \\
\hline Monounsaturated fat (\% energy) & 11.5 & $11 \cdot 2,11 \cdot 7$ & $11 \cdot 7$ & $11 \cdot 4,12 \cdot 0$ & $11 \cdot 2$ & $10 \cdot 9,11.4$ & $11 \cdot 3$ & $11 \cdot 1,11 \cdot 6$ \\
\hline Polyunsaturated fat (g) & $12 \cdot 8$ & $12 \cdot 1,13 \cdot 5$ & $15 \cdot 9$ & $15 \cdot 1,16 \cdot 6$ & $13 \cdot 3$ & $12 \cdot 6,14 \cdot 0$ & $12 \cdot 7$ & $12 \cdot 0,13 \cdot 4$ \\
\hline Polyunsaturated fat (\% energy) & $6 \cdot 3$ & $6 \cdot 0,6 \cdot 6$ & $7 \cdot 4$ & $7 \cdot 1,7 \cdot 7$ & 6.4 & $6 \cdot 1,6 \cdot 7$ & $6 \cdot 2$ & $5 \cdot 9,6 \cdot 4$ \\
\hline Carbohydrate (g) & $252 \cdot 3$ & $246 \cdot 0,258 \cdot 7$ & 258.5 & $251 \cdot 7,265 \cdot 2$ & $256 \cdot 3$ & $250 \cdot 0,262 \cdot 6$ & 252.4 & $246 \cdot 0,258 \cdot 7$ \\
\hline Carbohydrate (\% energy) & $52 \cdot 1$ & $51 \cdot 5,52 \cdot 7$ & 50.9 & $50 \cdot 2,51 \cdot 5$ & 53.0 & $52 \cdot 3,53 \cdot 6$ & 51.9 & $51 \cdot 3,52 \cdot 5$ \\
\hline Sugars $(g)$ & $116 \cdot 1$ & $111 \cdot 9,120 \cdot 3$ & $101 \cdot 2$ & $96 \cdot 7,105 \cdot 7$ & $112 \cdot 0$ & $107 \cdot 8,116 \cdot 2$ & $110 \cdot 8$ & $106 \cdot 6,115 \cdot 0$ \\
\hline Sugars (\% energy) & $23 \cdot 8$ & $23 \cdot 2,24 \cdot 5$ & $19 \cdot 8$ & $19 \cdot 1,20 \cdot 5$ & 22.9 & $22 \cdot 3,23 \cdot 6$ & $22 \cdot 7$ & $22 \cdot 0,23 \cdot 3$ \\
\hline Starch, dextrins (g) & $134 \cdot 2$ & $130 \cdot 2,138 \cdot 3$ & 154.9 & $150 \cdot 6,159 \cdot 2$ & 141.6 & $137 \cdot 6,145 \cdot 6$ & $139 \cdot 4$ & $135 \cdot 3,143 \cdot 4$ \\
\hline Starch, dextrins (\% energy) & $27 \cdot 8$ & $27 \cdot 2,28 \cdot 4$ & $30 \cdot 6$ & $30 \cdot 0,31 \cdot 3$ & $29 \cdot 4$ & $28 \cdot 8,30 \cdot 0$ & $28 \cdot 7$ & $28 \cdot 1,29 \cdot 3$ \\
\hline Total NSP $(\mathrm{g})$ & 11.8 & $11 \cdot 3,12 \cdot 2$ & $12 \cdot 8$ & $12 \cdot 3,13 \cdot 3$ & 11.0 & $10 \cdot 5,11.4$ & 11.5 & $11 \cdot 1,12 \cdot 0$ \\
\hline Protein $(\mathrm{g})$ & $57 \cdot 3$ & $55 \cdot 5,59 \cdot 2$ & $65 \cdot 7$ & $63 \cdot 7,67 \cdot 6$ & $60 \cdot 2$ & $58 \cdot 4,62 \cdot 1$ & 61.9 & $60 \cdot 0,63 \cdot 7$ \\
\hline Protein (\% energy) & $12 \cdot 7$ & $12 \cdot 4,13 \cdot 0$ & $13 \cdot 6$ & $13 \cdot 3,14 \cdot 0$ & $13 \cdot 3$ & $13 \cdot 0,13 \cdot 6$ & $13 \cdot 6$ & $13 \cdot 3,13 \cdot 9$ \\
\hline Vitamin $B_{12}(\mu \mathrm{g}) \dagger$ & $2 \cdot 8$ & $2 \cdot 6,3 \cdot 0$ & 2.9 & $2 \cdot 7,3 \cdot 1$ & 3.0 & $2 \cdot 8,3 \cdot 3$ & $3 \cdot 1$ & $2 \cdot 9,3 \cdot 3$ \\
\hline Folate $(\mu \mathrm{g}) \dagger$ & 204 & 196,212 & 196 & 187,204 & 200 & 192, 208 & 197 & 189, 205 \\
\hline Vitamin C (mg)† & $85 \cdot 1$ & $78 \cdot 9,91 \cdot 8$ & 73.9 & $68 \cdot 1,80 \cdot 1$ & 85.9 & $79 \cdot 6,92 \cdot 6$ & $80 \cdot 2$ & $74 \cdot 3,86 \cdot 5$ \\
\hline Vitamin $D(\mu \mathrm{g}) \dagger$ & 1.9 & $1 \cdot 8,2 \cdot 1$ & 1.4 & $1 \cdot 2,1.5$ & 1.7 & $1 \cdot 6,1 \cdot 8$ & 1.6 & $1.5,1.7$ \\
\hline $\mathrm{Ca}(\mathrm{mg}) \dagger$ & 742 & 712,773 & 699 & 669,731 & 693 & 665,722 & 725 & 695,755 \\
\hline $\mathrm{Fe}(\mathrm{mg}) \dagger$ & 8.7 & $8 \cdot 4,9 \cdot 0$ & 9.4 & $9 \cdot 0,9 \cdot 7$ & 8.6 & $8 \cdot 3,8.9$ & $8 \cdot 7$ & $8 \cdot 4,9 \cdot 0$ \\
\hline Haem Fe $(\mathrm{mg}) \dagger$ & 0.15 & $0.13,0.19$ & 0.12 & $0.10,0.15$ & 0.20 & $0.17,0.25$ & 0.19 & $0.16,0.23$ \\
\hline
\end{tabular}

* Means adjusted for age in quartiles, sex, day of week, month, interviewer and school (random effect).

† Geometric mean.

Patterns of nutritional composition among the white European group and the 'other ethnicity' group were very similar. There were, however, marked differences in nutritional composition between South Asians and white Europeans, summarised in Table 3, and between black African-Caribbeans and white Europeans, summarised in Table 4.

\section{South Asians: comparisons with white Europeans}

Total energy intake was higher among South Asians, mainly reflecting higher intakes of total fat and total protein, which contributed to greater percentages of total energy intake than in white Europeans. Saturated fat provided a lower percentage of energy and polyunsaturated fat a higher percentage of energy among South Asians, but the contribution of monounsaturated fat was not markedly different. Total carbohydrate intake was similar, but total carbohydrate intake as a percentage of energy was lower. This particularly reflected lower intakes of sugars (both in absolute amounts and as percentages of energy) while starch intakes were higher. NSP intake was higher among South Asians. Intakes of several micronutrients, including vitamin $\mathrm{C}$, vitamin $\mathrm{D}$ and $\mathrm{Ca}$ were lower, though total $\mathrm{Fe}$ intake was higher.

Differences in nutritional composition between white Europeans and South Asians specifically of Indian, Pakistani and Bangladeshi origin are shown in Table 3. The higher total energy intakes, the higher proportions of energy obtained from fat, polyunsaturated fat, starch and protein and the lower proportions of energy from saturated fat and sugars observed in all South Asians were particularly marked among the Bangladeshi children (who also showed particularly high intakes of monounsaturated fat) and to a lesser extent the Pakistani children; Indian children generally showed nutrient intake patterns closer to those of white Europeans. Similarly, low micronutrient intakes (particularly of vitamin $\mathrm{C}$ and $\mathrm{Ca}$ ) were particularly marked among Bangladeshi children, who also had lower folate intakes. Bangladeshi children had lower NSP intake than white Europeans, while Indian and Pakistani children had higher intakes.

\section{Black African-Caribbeans: comparisons with white Europeans}

Total energy intake was similar among black African-Caribbean and white European children (Tables 2 and 4). However, black African-Caribbeans derived lower percentages of energy from total and saturated fat and higher percentages of energy from protein and carbohydrate. Monounsaturated fat and polyunsaturated fat intakes did not differ markedly from white European intakes. Intake of starch was markedly higher while intakes of NSP and sugars tended to be lower. Black African-Caribbean children had lower intakes of $\mathrm{Ca}$ and vitamin D but a higher intake of haem Fe.

Differences in nutritional composition between white Europeans and black African-Caribbeans specifically of African and Caribbean origin are shown in Table 4. Black African children had particularly low percentages of energy intake from total and saturated fat and obtained a higher 
Table 3. Ethnic differences in nutritional composition of children's diets: South Asians compared with white Europeans* (Mean differences or percentage differences and $95 \%$ confidence intervals)

\begin{tabular}{|c|c|c|c|c|c|c|c|c|c|c|}
\hline & \multicolumn{10}{|c|}{ South Asian } \\
\hline & & & & & & South As & an subgroups & & & \\
\hline & \multicolumn{3}{|c|}{$\begin{array}{l}\text { All South Asian } \\
\text { ( } n \text { 558; } 264 \text { boys) }\end{array}$} & \multicolumn{2}{|c|}{$\begin{array}{c}\text { Indian } \\
\text { ( } n \text { 137; } 73 \text { boys) }\end{array}$} & \multicolumn{2}{|c|}{$\begin{array}{c}\text { Pakistani } \\
\text { (n 210; } 100 \text { boys) }\end{array}$} & \multicolumn{2}{|c|}{$\begin{array}{c}\text { Bangladeshi } \\
\text { ( } n \text { 175; } 75 \text { boys) }\end{array}$} & \multirow[b]{2}{*}{$\begin{array}{l}P \text { (no difference } \\
\text { between South } \\
\text { Asian subgroups) }\end{array}$} \\
\hline & $\begin{array}{c}\text { Mean } \\
\text { difference }\end{array}$ & $95 \% \mathrm{Cl}$ & $\begin{array}{c}P \\
\text { (no difference) }^{\star}\end{array}$ & $\begin{array}{c}\text { Mean } \\
\text { difference }\end{array}$ & $95 \% \mathrm{Cl}$ & $\begin{array}{c}\text { Mean } \\
\text { difference }\end{array}$ & $95 \% \mathrm{Cl}$ & $\begin{array}{c}\text { Mean } \\
\text { difference }\end{array}$ & $95 \% \mathrm{Cl}$ & \\
\hline Energy (kJ) & 408 & 147,669 & 0.002 & 204 & $-202,610$ & 559 & 205,913 & 482 & 104,859 & 0.25 \\
\hline Energy (kcal) & 97 & 35,159 & 0.002 & 48 & $-49,145$ & 133 & 49,218 & 115 & 25,205 & 0.25 \\
\hline Fat $(\mathrm{g})$ & $6 \cdot 1$ & $2 \cdot 8,9.4$ & $<0.001$ & $2 \cdot 7$ & $-2 \cdot 4,7.9$ & $7 \cdot 6$ & $3 \cdot 1,12 \cdot 0$ & 8.5 & $3 \cdot 8,13 \cdot 3$ & $0 \cdot 10$ \\
\hline Fat (\% energy) & 1.0 & $0.2,1 \cdot 8$ & 0.01 & 0.4 & $-0.8,1.7$ & 1.0 & $-0.1,2 \cdot 1$ & 1.8 & $0.6,2 \cdot 9$ & 0.23 \\
\hline Saturated fat $(\mathrm{g})$ & 0.1 & $-1.3,1.5$ & 0.85 & -0.1 & $-2 \cdot 3,2 \cdot 1$ & 1.4 & $-0.5,3.3$ & -0.9 & $-3 \cdot 0,1 \cdot 1$ & 0.17 \\
\hline Saturated fat (\% energy) & -0.6 & $-1 \cdot 0,-0.1$ & 0.01 & -0.3 & $-0.9,0.4$ & -0.3 & $-0.8,0.3$ & $-1 \cdot 2$ & $-1.8,-0.6$ & 0.03 \\
\hline Monounsaturated fat $(\mathrm{g})$ & 1.8 & $0.6,3 \cdot 0$ & 0.003 & -0.3 & $-2 \cdot 1,1 \cdot 6$ & 1.9 & $0.3,3.5$ & $4 \cdot 2$ & $2 \cdot 4,5 \cdot 9$ & $<0.001$ \\
\hline Monounsaturated fat ( $\%$ energy) & 0.2 & $-0.1,0.6$ & 0.19 & -0.4 & $-0.9,0.2$ & 0.0 & $-0.5,0.5$ & 1.2 & $0.7,1.7$ & $<0.0001$ \\
\hline Polyunsaturated fat $(\mathrm{g})$ & $3 \cdot 0$ & $2 \cdot 1,4 \cdot 0$ & $<0.0001$ & $2 \cdot 1$ & $0.6,3.6$ & $2 \cdot 7$ & $1.4,4.0$ & 4.5 & $3.1,5.9$ & 0.02 \\
\hline Polyunsaturated fat (\% energy) & $1 \cdot 1$ & $0.7,1.5$ & $<0.0001$ & 0.8 & $0.2,1.4$ & 0.8 & $0.2,1.3$ & 1.8 & $1 \cdot 2,2 \cdot 3$ & 0.01 \\
\hline Carbohydrate $(\mathrm{g})$ & $6 \cdot 1$ & $-2 \cdot 7,14.9$ & 0.17 & 6.9 & $-6 \cdot 7,20 \cdot 6$ & $10 \cdot 4$ & $-1 \cdot 5,22 \cdot 3$ & $2 \cdot 0$ & $-10 \cdot 7,14 \cdot 7$ & 0.61 \\
\hline Carbohydrate (\% energy) & $-1 \cdot 2$ & $-2 \cdot 1,-0.4$ & 0.005 & 0.3 & $-1 \cdot 0,1 \cdot 6$ & -1.3 & $-2.5,-0.2$ & -2.5 & $-3 \cdot 8,-1 \cdot 3$ & 0.003 \\
\hline Sugars $(g)$ & -14.9 & $-20.5,-9.2$ & $<0.0001$ & -14.9 & $-23 \cdot 7,-6 \cdot 1$ & $-5 \cdot 8$ & $-13 \cdot 5,1 \cdot 9$ & -24.5 & $-32 \cdot 7,-16 \cdot 3$ & $<0.001$ \\
\hline Sugars (\% energy) & -4.0 & $-4 \cdot 9,-3 \cdot 1$ & $<0.0001$ & -3.3 & $-4 \cdot 7,-2 \cdot 0$ & -2.6 & $-3 \cdot 8,-1.5$ & $-6 \cdot 2$ & $-7.5,-4.9$ & $<0.0001$ \\
\hline Starch, dextrins $(\mathrm{g})$ & 20.7 & $15 \cdot 2,26 \cdot 1$ & $<0.0001$ & 21.5 & $13 \cdot 1,30 \cdot 0$ & $16 \cdot 1$ & $8 \cdot 7,23.5$ & 25.9 & $18 \cdot 1,33.8$ & 0.09 \\
\hline Starch, dextrins (\% energy) & $2 \cdot 8$ & $2 \cdot 0,3 \cdot 6$ & $<0.0001$ & 3.6 & $2 \cdot 4,4 \cdot 8$ & 1.4 & $0.4,2.5$ & 3.6 & $2 \cdot 4,4 \cdot 7$ & $<0.001$ \\
\hline Total NSP $(\mathrm{g})$ & $1 \cdot 1$ & $0.5,1 \cdot 7$ & $<0.001$ & $3 \cdot 7$ & $2 \cdot 8,4 \cdot 6$ & 1.5 & $0 \cdot 7,2 \cdot 3$ & $-1 \cdot 3$ & $-2.2,-0.5$ & $<0.0001$ \\
\hline Protein $(\mathrm{g})$ & $8 \cdot 3$ & $5 \cdot 8,10 \cdot 9$ & $<0.0001$ & 1.3 & $-2 \cdot 6,5 \cdot 2$ & $9 \cdot 6$ & $6 \cdot 2,13 \cdot 0$ & 13.7 & $10 \cdot 0,17 \cdot 3$ & $<0.0001$ \\
\hline Protein (\% energy) & 0.9 & $0.5,1.3$ & $<0.0001$ & -0.4 & $-1 \cdot 0,0.3$ & 1.0 & $0.4,1.5$ & 1.9 & $1 \cdot 3,2 \cdot 5$ & $<0.0001$ \\
\hline Vitamin $B_{12}(\mu \mathrm{g}) \dagger$ & 3.6 & $-5 \cdot 8,13.9$ & 0.47 & -7.0 & $-19 \cdot 7,7 \cdot 8$ & $16 \cdot 4$ & $2 \cdot 3,32 \cdot 4$ & 1.0 & $-12 \cdot 0,15 \cdot 9$ & 0.03 \\
\hline Folate $(\mu \mathrm{g}) \dagger$ & -4.2 & $-9 \cdot 1,1 \cdot 1$ & 0.12 & 0.1 & $-7 \cdot 8,8 \cdot 7$ & -1.6 & $-8 \cdot 4,5 \cdot 8$ & $-10 \cdot 6$ & $-17 \cdot 2,-3 \cdot 4$ & 0.05 \\
\hline Vitamin C $(\mathrm{mg}) \dagger$ & $-13 \cdot 2$ & $-21 \cdot 6,-3 \cdot 8$ & 0.01 & -6.0 & $-19 \cdot 7,10 \cdot 1$ & $-10 \cdot 6$ & $-22 \cdot 2,2 \cdot 6$ & $-21 \cdot 7$ & $-32 \cdot 4,-9 \cdot 2$ & 0.12 \\
\hline Vitamin $D(\mu \mathrm{g}) \dagger$ & -29.9 & $-37 \cdot 8,-21 \cdot 1$ & $<0.0001$ & -29.9 & $-41 \cdot 7,-15 \cdot 7$ & -25.9 & $-36 \cdot 9,-12 \cdot 9$ & $-36 \cdot 8$ & $-46 \cdot 8,-25 \cdot 0$ & 0.29 \\
\hline $\mathrm{Ca}(\mathrm{mg}) \dagger$ & -5.8 & $-11 \cdot 0,-0.3$ & 0.04 & 1.6 & $-6 \cdot 8,10 \cdot 8$ & $2 \cdot 1$ & $-5 \cdot 3,10 \cdot 1$ & $-18 \cdot 8$ & $-25 \cdot 1,-12 \cdot 0$ & $<0.0001$ \\
\hline $\mathrm{Fe}(\mathrm{mg}) \dagger$ & $7 \cdot 4$ & $2 \cdot 7,12 \cdot 4$ & 0.002 & 10.4 & $2 \cdot 9,18 \cdot 5$ & $8 \cdot 8$ & $2 \cdot 4,15 \cdot 7$ & 4.2 & $-2 \cdot 4,11 \cdot 2$ & 0.37 \\
\hline Haem Fe $(m g) \dagger$ & $-19 \cdot 6$ & $-36 \cdot 8,2 \cdot 1$ & 0.07 & $-71 \cdot 7$ & $-80.4,-59.3$ & $-12 \cdot 2$ & $-36 \cdot 4,21 \cdot 3$ & 53.8 & $8 \cdot 9,117 \cdot 0$ & $<0.0001$ \\
\hline
\end{tabular}

${ }^{*}$ Differences (South Asian minus white European in all cases) are adjusted for age in quartiles, sex, day of week, month, interviewer and school (random effect). Thirty-six South Asians could not be classified specifically as Indian, Pakistani or Bangladeshi and are therefore omitted from South Asian subgroup analysis.

†Percentage difference. 
Table 4. Ethnic differences in nutritional composition of children's diets: black African-Caribbeans compared with white Europeans* (Mean differences or percentage differences and $95 \%$ confidence intervals)

\begin{tabular}{|c|c|c|c|c|c|c|c|c|}
\hline & \multicolumn{8}{|c|}{ Black African-Caribbean } \\
\hline & & & & & Black African-Ca & bean subgroups & & \multirow{3}{*}{$\begin{array}{c}P \text { (no difference } \\
\text { between black subgroups) }\end{array}$} \\
\hline & \multicolumn{3}{|c|}{$\begin{array}{c}\text { All black } \\
\text { ( } n \text { 560; } 261 \text { boys) }\end{array}$} & \multicolumn{2}{|c|}{$\begin{array}{l}\text { Black African } \\
\text { ( } n \text { 309; } 136 \text { boys) }\end{array}$} & \multicolumn{2}{|c|}{$\begin{array}{l}\text { Black Caribbean } \\
\text { ( } n 210 ; 105 \text { boys) }\end{array}$} & \\
\hline & Mean difference & $95 \% \mathrm{Cl}$ & $P{\text { (no difference })^{\star}}^{*}$ & Mean difference & $95 \% \mathrm{Cl}$ & Mean difference & $95 \% \mathrm{Cl}$ & \\
\hline Energy (kJ) & 31 & $-217,279$ & 0.81 & 76 & $-217,369$ & 25 & $-307,358$ & 0.73 \\
\hline Energy (kcal) & 7 & $-52,66$ & 0.82 & 17 & $-53,87$ & 6 & $-74,85$ & 0.74 \\
\hline Fat $(\mathrm{g})$ & -1.9 & $-5 \cdot 0,1 \cdot 3$ & 0.24 & $-2 \cdot 8$ & $-6.5,0.9$ & -0.5 & $-4 \cdot 7,3 \cdot 7$ & 0.34 \\
\hline Fat (\% energy) & $-1 \cdot 3$ & $-2 \cdot 1,-0.5$ & $<0.001$ & $-2 \cdot 0$ & $-2 \cdot 9,-1 \cdot 1$ & $-0 \cdot 6$ & $-1 \cdot 6,0 \cdot 4$ & 0.01 \\
\hline Saturated fat $(\mathrm{g})$ & $-2 \cdot 1$ & $-3.5,-0.8$ & 0.002 & $-2 \cdot 7$ & $-4 \cdot 3,-1 \cdot 1$ & -1.3 & $-3.1,0.5$ & 0.17 \\
\hline Saturated fat (\% energy) & $-1 \cdot 2$ & $-1 \cdot 6,-0.8$ & $<0.0001$ & -1.5 & $-2 \cdot 0,-1 \cdot 0$ & -0.8 & $-1.4,-0.3$ & 0.02 \\
\hline Monounsaturated fat (g) & -0.5 & $-1 \cdot 7,0 \cdot 6$ & 0.37 & -0.9 & $-2 \cdot 2,0 \cdot 5$ & 0.0 & $-1.5,1.5$ & 0.31 \\
\hline Monounsaturated fat (\% energy) & -0.3 & $-0.7,0.0$ & 0.07 & -0.5 & $-0.9,-0.1$ & -0.1 & $-0.5,0.4$ & 0.07 \\
\hline Polyunsaturated fat $(\mathrm{g})$ & 0.5 & $-0.5,1.4$ & 0.32 & 0.4 & $-0.7,1 \cdot 4$ & 0.4 & $-0.8,1.6$ & 0.95 \\
\hline Polyunsaturated fat (\% energy) & 0.1 & $-0.2,0.5$ & 0.54 & 0.0 & $-0.4,0.4$ & 0.1 & $-0.4,0.6$ & 0.58 \\
\hline Carbohydrate (g) & 4.0 & $-4 \cdot 4,12 \cdot 3$ & 0.35 & $9 \cdot 2$ & $-0.6,19.0$ & -0.2 & $-11 \cdot 3,11 \cdot 0$ & 0.11 \\
\hline Carbohydrate (\% energy) & 0.8 & $0 \cdot 0,1 \cdot 7$ & 0.05 & 1.6 & $0 \cdot 7,2 \cdot 6$ & 0.0 & $-1 \cdot 1,1 \cdot 1$ & 0.01 \\
\hline Sugars $(g)$ & $-4 \cdot 1$ & $-9 \cdot 4,1 \cdot 3$ & 0.14 & $-4 \cdot 1$ & $-10 \cdot 4,2 \cdot 2$ & $-4 \cdot 2$ & $-11 \cdot 4,2 \cdot 9$ & 0.93 \\
\hline Sugars (\% energy) & -0.9 & $-1 \cdot 7,-0 \cdot 1$ & 0.04 & $-1 \cdot 0$ & $-2 \cdot 0,0 \cdot 0$ & -0.9 & $-2 \cdot 0,0 \cdot 2$ & 0.92 \\
\hline Starch, dextrins $(\mathrm{g})$ & $7 \cdot 4$ & $2 \cdot 2,12 \cdot 6$ & 0.01 & $12 \cdot 6$ & $6 \cdot 5,18 \cdot 7$ & 3.8 & $-3 \cdot 1,10 \cdot 7$ & 0.02 \\
\hline Starch, dextrins (\% energy) & 1.6 & $0 \cdot 9,2 \cdot 3$ & $<0.0001$ & 2.5 & $1 \cdot 6,3 \cdot 3$ & 0.8 & $-0 \cdot 1,1 \cdot 8$ & 0.003 \\
\hline Total NSP (g) & -0.8 & $-1.4,-0.3$ & 0.004 & -0.7 & $-1 \cdot 3,-0 \cdot 1$ & -0.8 & $-1.5,0.0$ & 0.90 \\
\hline Protein $(\mathrm{g})$ & 2.9 & $0.5,5 \cdot 3$ & 0.02 & 3.1 & $0.3,5.9$ & 3.0 & $-0.2,6 \cdot 2$ & 0.91 \\
\hline Protein (\% energy) & 0.6 & $0.2,1 \cdot 0$ & 0.003 & 0.5 & $0 \cdot 1,1 \cdot 0$ & 0.6 & $0 \cdot 1,1 \cdot 1$ & 0.85 \\
\hline Vitamin $B_{12}(\mu \mathrm{g}) \dagger$ & 9.7 & $0 \cdot 2,20 \cdot 1$ & 0.04 & 14.4 & $2 \cdot 9,27 \cdot 3$ & 5.9 & $-6 \cdot 1,19.5$ & 0.21 \\
\hline Folate $(\mu \mathrm{g}) \dagger$ & $-2 \cdot 2$ & $-7 \cdot 0,2 \cdot 9$ & 0.39 & 3.6 & $-2 \cdot 4,9.9$ & -5.9 & $-12 \cdot 0,0 \cdot 6$ & 0.01 \\
\hline Vitamin C $(\mathrm{mg}) \dagger$ & 0.9 & $-8 \cdot 3,11 \cdot 1$ & 0.85 & 1.5 & $-9 \cdot 4,13 \cdot 7$ & 1.2 & $-11 \cdot 1,15 \cdot 1$ & 0.96 \\
\hline Vitamin D $(\mu \mathrm{g}) \dagger$ & $-12 \cdot 1$ & $-21.5,-1.6$ & 0.03 & $-14 \cdot 2$ & $-24 \cdot 9,-2 \cdot 1$ & -9.4 & $-22 \cdot 1,5 \cdot 3$ & 0.53 \\
\hline $\mathrm{Ca}(\mathrm{mg}) \dagger$ & $-6 \cdot 6$ & $-11.4,-1.5$ & 0.012 & -4.1 & $-9 \cdot 9,2 \cdot 1$ & $-8 \cdot 0$ & $-14 \cdot 3,-1 \cdot 3$ & 0.29 \\
\hline $\mathrm{Fe}(\mathrm{mg}) \dagger$ & $-1 \cdot 2$ & $-5 \cdot 3,3 \cdot 2$ & 0.59 & $2 \cdot 0$ & $-3 \cdot 0,7 \cdot 4$ & $-3 \cdot 2$ & $-8 \cdot 6,2 \cdot 6$ & 0.09 \\
\hline Haem Fe $(\mathrm{mg}) \dagger$ & $32 \cdot 0$ & $5 \cdot 5,65 \cdot 1$ & 0.015 & $22 \cdot 8$ & $-5.4,59.3$ & $52 \cdot 1$ & $13 \cdot 1,104 \cdot 5$ & 0.22 \\
\hline
\end{tabular}

${ }^{\star}$ Differences (black African-Caribbean minus white European in all cases) are adjusted for age in quartiles, sex, day of week, month, interviewer and school (random effect). Forty-one black African-Caribbeans could not be classified specifically as black African or black Caribbean and are therefore omitted from black African-Caribbean subgroup analysis.

$\dagger$ Percentage difference. 
percentage of energy from carbohydrate (particularly from starch). They had markedly higher folate intakes than black Caribbean children. Black Caribbean children appeared to have fat and carbohydrate intakes much closer to those of white Europeans.

The patterns of ethnic differences described between South Asians, black African-Caribbeans and white Europeans were similar in boys and girls. In further analyses, the ethnic differences were not affected by adjustment for socio-economic status based on parental occupation or parental education. Additional adjustment for height (a strong proxy marker of socio-economic status ${ }^{(46)}$ ) also had no material effect on the results. The replacement of the white European ethnic group by white British children ( $n$ 372) had little effect on the pattern of ethnic differences observed. In sensitivity analyses excluding under-reporters ( $n$ 476) and over-reporters ( $n$ 149), the pattern of ethnic differences in nutrient intakes observed was not materially altered.

\section{Discussion}

The results of this detailed study of 9- to 10-year-old children suggest that there are marked ethnic differences in nutritional composition in the UK. Compared with white Europeans, children of South Asian origin appeared to have higher intakes of total energy, total and polyunsaturated fat, protein and starch (both absolute and as proportions of energy), while their intake of sugars and many micronutrients appeared lower. These differences were particularly marked among the children of Bangladeshi origin. Black African-Caribbean children, in contrast, had lower intakes of total and saturated fat (both absolute and as proportions of energy). Intakes of sugars and NSP were also lower, while intakes of protein and starch were higher. $\mathrm{Ca}$ intake was lower and $\mathrm{Fe}$ intake higher. The differences in fat and starch intake were particularly marked among black African children.

\section{Agreement with previous studies}

The possibility that energy intakes are higher in South Asians, though consistent with one previous report in adults ${ }^{(47)}$, has not been observed in several other studies in adults, which showed little or no difference in energy intakes between South Asians and white Europeans ${ }^{(20-22)}$. The higher fat intakes (in particular, higher percentage of energy derived from polyunsaturated fats) and lower intakes of micronutrients observed among South Asians in the present study are consistent with differences both in fat intake and vitamin $\mathrm{C}$ intake reported in earlier studies in adults ${ }^{(20-24,48)}$. However, thehigher saturated fat intakes reported in a recent study of South Asian adults were not confirmed here ${ }^{(23)}$. The lower fat intakes (in particular, the lower percentage of energy derived from saturated fat) reported for black African-Caribbean children are consistent with the differences in fat intake reported in earlier studies in adults ${ }^{(22)}$. The nutrient intake patterns observed in the present study closely reflect ethnic differences in food group items consumed by the children. In preliminary analyses in comparison with white Europeans, South Asian children derived much higher proportions of energy from pasta and rice, fats, milk and 'other vegetables' and lower proportions from potatoes, leafy vegetables, breakfast cereals, cheese and yoghurts, and fruit and nuts. Black AfricanCaribbean children, in contrast, obtained greater proportions of energy from meat, milk, pasta and rice and 'other vegetables' in their diets and much less from potatoes, breads, fats, fish, cheese and yoghurt and fruit and nuts. These differences could well help to explain both macronutrient and micronutrient intakes between these groups.

\section{Strengths and limitations of the present study}

The strengths and limitations of the study merit consideration. The present study was based in three cities which together account for more than two-thirds of South Asians and black African-Caribbeans living in the UK and specifically included South Asian children of Indian, Pakistani and Bangladeshi origin and black African-Caribbean children of black African and black Caribbean origin. Comparisons of nutritional composition were strengthened by being conducted on a withinschool basis, to limit the scope for confounding, especially for socio-economic factors. Although response rates were moderate (probably reflecting the appreciable social deprivation of the population studied), response rates did not differ markedly by ethnic group and are unlikely to account for the ethnic differences in dietary composition observed. A single structured $24 \mathrm{~h}$ recall assessment was used because it has been previously shown to provide a valid and unbiased estimate of usual dietary intake in individuals, making it particularly suitable for group-level comparisons in children of this age ${ }^{(32-34)}$ as well as in adults ${ }^{(31)}$. The method is more suitable than food-frequency approaches for this age group ${ }^{(49)}$, does not demand high levels of literacy and is particularly suitable for large-scale surveys ${ }^{(35)}$. The use of direct interviews without involving a carer has the advantage of providing a more complete picture of nutrient intake (including foods eaten outside the home) which is not biased by the presence of the carer ${ }^{(33)}$. The validity of the estimates of nutrient intakes obtained using the $24 \mathrm{~h}$ recall method in the present study is supported by several observations. First, estimates of all nutrient intakes were positively correlated with estimates obtained using prospective $4 \mathrm{~d}$ dietary diaries in a sub-study in 500 children (AS Donin, CM Nightingale, CG Owen, AR Rudnicka, MC McNamara, CJ Prynne, AM Stephen, DG Cook and PH Whincup, unpublished results). Second, overall intakes of both macronutrients and micronutrients in the present study are close to those of 7- to 10-year-old children in the 2000 National Diet and Nutrition Survey $\left(\right.$ NDNS) ${ }^{(50)}$, which used prospective, $7 \mathrm{~d}$ weighed food diaries to assess food intakes; the prevalence of under-reporting of total energy intake was also similar to (though slightly lower than) under-reporting rates reported in the NDNS for the 7to 10-year age group (29\% for boys and $27 \%$ for girls). Third, the patterns of ethnic differences in nutrient intakes in the present study are substantially consistent with those reported in previous studies in adults ${ }^{(20,22,47)}$. Although the present study was primarily based on nutrient intake assessments for weekdays during school terms, these account for at least half the days in the year at this age and are likely to be reasonably representative; the exclusion of holiday periods is likely to have led to underestimation rather than overestimation of the extent of ethnic differences in nutrient intakes. Although the reporting of dietary intake (particularly energy 
intake) is known to be influenced by participant characteristics (particularly body weight), there is little evidence that reporting of nutrient intakes varies by ethnic group ${ }^{(22)}$. The higher reported energy intakes observed in South Asian children in the present study could reflect true differences, but they may also reflect a specific problem in the assessment of portion sizes in this group. In the present study, most South Asian children reported a distinctive pattern of meal intake, having two full meals in the later part of the day (one immediately on returning home, with a family meal later in the evening). Further investigation will be needed to resolve whether the meal pattern has resulted in overestimation of total energy intake, though it would not be expected to affect estimates of macronutrient intakes expressed as proportions of total energy.

\section{Implications of ethnic differences in nutritional composition for future chronic disease risk}

The persistence of the differences in nutritional composition between South Asian and white European children observed in the present study could have important consequences for longer-term risks of obesity, CHD and type 2 diabetes among the South Asians. If substantiated in further studies, the higher total energy intakes observed for South Asian children, primarily derived from fat and protein, may induce positive energy balance and be responsible for appreciable increases in risks of longer-term overweight and obesity ${ }^{(51,52)}$, particularly at the lower levels of physical activity reported in South Asians ${ }^{(53)}$. Long-term increases in overweight and obesity from childhood onwards may themselves increase risks of CHD and type 2 diabetes $^{(54)}$. The high average total fat intake among South Asian children (accounting for about $36 \%$ of energy intake) suggests that most of these children exceed the Committee on Medical Aspects of Food and Nutrition Policy (COMA) recommendation for a maximum of $35 \%$ of energy to be derived from fat. The higher overall fat intake may have implications for long-term risks of type 2 diabetes and $\mathrm{CHD}^{(55)}$; high polyunsaturated fat intakes have been associated with higher fasting serum insulin levels in young Asian Indians ${ }^{(39)}$. The higher intakes of starch in South Asians may enhance glycaemic response, reducing insulin sensitivity and increasing TAG levels ${ }^{(56)}$. The lower levels of micronutrient intakes observed in South Asians, particularly of vitamin C, vitamin $\mathrm{D}$ and $\mathrm{Ca}$, could also affect long-term disease risks. In adults, vitamin $C$ intake shows a graded inverse relationship with risks of type 2 diabetes and CVD, even within the normal range ${ }^{(57,58)}$. Evidence suggests that low vitamin $D$ intake and (in particular) low circulating levels of vitamin D and Ca may be associated with increased risks of insulin resistance, type 2 diabetes and $\mathrm{CHD}^{(59-61)}$. The particularly marked nutritional differences between Bangladeshi and white European children are consistent with the markedly higher risks of type 2 diabetes and CHD among UK Bangladeshi adults ${ }^{(2,3)}$. It is also possible that genetic factors modify the influence of nutrient intakes on risks of CVD and type 2 diabetes, and that Asian populations could be particularly susceptible ${ }^{(62)}$, though this has still to be established.

For the black African-Caribbeans, the lower proportions of energy derived from total and saturated fat would be expected to lead to a favourable lipid profile, particularly lower LDL-cholesterol, which would be expected to reduce the rate of coronary atherosclerotic plaque development and $\mathrm{CHD}^{(63)}$. Such findings are consistent with the lower long-term risks of CHD observed in black African-Caribbean adults ${ }^{(2,3,5)}$. However, the finding that total and saturated fat intakes were particularly low among black Africans, while those of black Caribbeans are closer to those of white Europeans, suggests that the diets of the black Caribbean population may have become increasingly westernised since migration, and that the protection against CHD previously experienced by black African-Caribbean adults ${ }^{(2,5)}$ may be increasingly concentrated among black Africans during the next generation. The effects of the slightly higher starch intakes among black African-Caribbeans remain uncertain, though they could have adverse long-term effects on insulin sensitivity ${ }^{(56)}$. The lower intakes of NSP among black African-Caribbean children could have important adverse long-term effects, impairing satiety signals and appetite control ${ }^{(16)}$, and increasing long-term risk of obesity ${ }^{(64)}$, diabetes ${ }^{(65,66)}$ and stroke ${ }^{(67)}$. The lower intakes of $\mathrm{Ca}$ and vitamin $\mathrm{D}$ (more so for $\mathrm{Ca}$ in African-Caribbeans, and for vitamin D in South Asians), particularly if reflected in low overall circulating vitamin D levels, could be relevant to increased longer-term risks of type 2 diabetes and $\operatorname{CVD}^{(59,61)}$.

\section{Conclusions}

There are substantial differences in the nutritional composition of the diets of children of South Asian, black AfricanCaribbean and white European origin living in the UK. The persistence of these dietary differences into adult life could contribute to the continuation of the current pattern of ethnic differences in chronic disease into the next generation.

\section{Acknowledgements}

The present study was supported by The Wellcome Trust (grant no. 068362/Z/02/Z) and the National Prevention Research Initiative (NPRI). The funding partners for the NPRI award were: British Heart Foundation; Cancer Research UK; Department of Health; Diabetes UK; Economic and Social Research Council; Medical Research Council; Health and Social Care Research and Development Office for Northern Ireland; Chief Scientist Officer, Scottish Government Health Directorate; Welsh Assembly Government; Food Standards Agency; World Cancer Research Fund. A. S. D. was supported by a British Heart Foundation PhD studentship (reference no. FS/08/022/24946).

We are grateful to Cathy McKay, Miranda Price, Andrea Wathern and Rahat Rafiq for their work on the organisation of the CHASE study and to the schools, parents and children who participated in the CHASE study. We would also like to thank the dietary assessment team at the Medical Research Council Human Nutrition Research Centre, particularly Sarah-Jane Flaherty and Jonathan Last for their diligent work in coding the dietary recalls from the study.

P. H. W., D. G. C., A. M. S. and A. S. D. were involved in study design; P. H. W., D. G. C., A. M. S., A. S. D., M. C. M., C. G. O. and C. J. P. were involved in data acquisition. C. M. N. and A. R. R. carried out statistical analyses; all authors contributed to data interpretation. A. S. D. and P. H. W. wrote the manuscript and all authors provided critical 
revision. P. H. W., D. G. C., A. M. S. and C. G. O. obtained funding and supervised the study.

None of the authors had a personal or financial conflict of interest.

\section{References}

1. Pomerleau J, McKeigue PM \& Chaturvedi N (1999) Factors associated with obesity in South Asian, Afro-Caribbean and European women. Int J Obes Relat Metab Disord 23, 25-33.

2. Wild SH, Fischbacher C, Brock A, et al. (2007) Mortality from all causes and circulatory disease by country of birth in England and Wales 2001-2003. J Public Health (Oxf) 29, 191-198.

3. Office for National Statistics (2004) Health Survey for England 2004: the Health of Minority Ethnic Groups. London: National Centre for Social Research.

4. McKeigue PM, Shah B \& Marmot MG (1991) Relation of central obesity and insulin resistance with high diabetes prevalence and cardiovascular risk in South Asians. Lancet 337, 382-386.

5. Wild S \& McKeigue P (1997) Cross sectional analysis of mortality by country of birth in England and Wales, 1970-92. BMJ 314, 705-710.

6. Chaturvedi N (2003) Ethnic differences in cardiovascular disease. Heart 89, 681-686.

7. Kuppuswamy VC \& Gupta S (2005) Excess coronary heart disease in South Asians in the United Kingdom. BMJ 330, $1223-1224$.

8. World Health Organization (2003) Diet, Nutrition and the Prevention of Chronic Diseases. Joint WHO/FAO Expert Consultation. WHO Technical Report Series no. 916. Geneva: WHO.

9. Vessby B, Unsitupa M, Hermansen K, et al. (2001) Substituting dietary saturated for monounsaturated fat impairs insulin sensitivity in healthy men and women: the KANWU Study. Diabetologia 44, 312-319.

10. Bo S, Menato G, Lezo A, et al. (2001) Dietary fat and gestational hyperglycaemia. Diabetologia 44, 972-978.

11. Hooper L, Summerbell CD, Higgins JP, et al. (2001) Dietary fat intake and prevention of cardiovascular disease: systematic review. BMJ 322, 757-763.

12. Mann J (2007) Dietary carbohydrate: relationship to cardiovascular disease and disorders of carbohydrate metabolism. Eur J Clin Nutr 61, Suppl. 1, S100-S111.

13. Fried SK \& Rao SP (2003) Sugars, hypertriglyceridemia, and cardiovascular disease. Am J Clin Nutr 78, 873S-880S.

14. Roche HM (1999) Dietary carbohydrates and triacylglycerol metabolism. Proc Nutr Soc 58, 201-207.

15. Ludwig DS, Pereira MA, Kroenke CH, et al. (1999) Dietary fiber, weight gain, and cardiovascular disease risk factors in young adults. JAMA 282, 1539-1546.

16. Anderson JW \& Hanna TJ (1999) Impact of nondigestible carbohydrates on serum lipoproteins and risk for cardiovascular disease. J Nutr 129, 1247S-1266S.

17. Ascherio A, Rimm EB, Hernan MA, et al. (1998) Intake of potassium, magnesium, calcium, and fiber and risk of stroke among US men. Circulation 98, 1198-1204.

18. Gale CR, Martyn CN, Winter PD, et al. (1995) Vitamin C and risk of death from stroke and coronary heart disease in cohort of elderly people. BMJ 310, 1563-1566.

19. Michos ED \& Melamed ML (2008) Vitamin D and cardiovascular disease risk. Curr Opin Clin Nutr Metab Care 11, $7-12$.

20. Miller GJ, Kotecha S, Wilkinson WH, et al. (1988) Dietary and other characteristics relevant for coronary heart disease in men of Indian, West Indian and European descent in London. Atherosclerosis 70, 63-72.
21. Sevak L, McKeigue PM \& Marmot MG (1994) Relationship of hyperinsulinemia to dietary intake in south Asian and European men. Am J Clin Nutr 59, 1069-1074.

22. Vyas A, Greenhalgh A, Cade J, et al. (2003) Nutrient intakes of an adult Pakistani, European and African-Caribbean community in inner city Britain. J Hum Nutr Diet 16, 327-337.

23. Anderson AS, Bush H, Lean M, et al. (2005) Evolution of atherogenic diets in South Asian and Italian women after migration to a higher risk region. J Hum Nutr Diet 18, 33-43.

24. Ness AR, Cappuccio FP, Atkinson RW, et al. (1999) Plasma vitamin $\mathrm{C}$ levels in men and women from different ethnic backgrounds living in England. Int J Epidemiol 28, 450-455.

25. Sharma S, Cade J, Riste L, et al. (1999) Nutrient intake trends among African-Caribbeans in Britain: a migrant population and its second generation. Public Health Nutr 2, 469-476.

26. Ehtisham S, Hattersley AT, Dunger DB, et al. (2004) First UK survey of paediatric type 2 diabetes and MODY. Arch Dis Child 89, 526-529.

27. Whincup PH, Gilg JA, Papacosta O, et al. (2002) Early evidence of ethnic differences in cardiovascular risk: cross sectional comparison of British South Asian and white children. BMJ 324, 635.

28. Whincup PH, Gilg JA, Owen CG, et al. (2005) British South Asians aged 13-16 years have higher fasting glucose and insulin levels than Europeans. Diabet Med 22, 1275-1277.

29. Wardle J, Brodersen NH, Cole TJ, et al. (2006) Development of adiposity in adolescence: five year longitudinal study of an ethnically and socioeconomically diverse sample of young people in Britain. BMJ 332, 1130-1135.

30. Saxena S, Ambler G, Cole TJ, et al. (2004) Ethnic group differences in overweight and obese children and young people in England: cross sectional survey. Arch Dis Child 89, $30-36$.

31. Gersovitz M, Madden JP \& Smiciklas-Wright H (1978) Validity of the 24-hr. dietary recall and seven-day record for group comparisons. J Am Diet Assoc 73, 48-55.

32. Frank GC, Berenson GS, Schilling PE, et al. (1977) Adapting the 24-hr. recall for epidemiologic studies of school children. $J$ Am Diet Assoc 71, 26-31.

33. Sobo EJ, Rock CL, Neuhouser ML, et al. (2000) Caretakerchild interaction during children's 24-hour dietary recalls: who contributes what to the recall record? J Am Diet Assoc 100, $428-433$.

34. Biro G, Hulshof KF, Ovesen L, et al. (2002) Selection of methodology to assess food intake. Eur J Clin Nutr 56, Suppl. 2, S25-S32.

35. Bingham SA \& Nelson M (1995) Assessment of food consumption and nutrient intake. In Design Concepts in Nutritional Epidemiology, pp. 153-191 [BM Margetts and M Nelson, editors]. Oxford: Oxford Medical Publications.

36. Cameron ME \& van Staveren WA (1988) Manual on Methodology for Food Consumption Studies. New York: Oxford Medical Publications.

37. Conway JM, Ingwersen LA \& Moshfegh AJ (2004) Accuracy of dietary recall using the USDA five-step multiple-pass method in men: an observational validation study. $J$ Am Diet Assoc 104, 595-603.

38. Nelson M, Atkinson M \& Meyer J (1997) A Photographic Atlas of Food Portion Sizes. London: MAFF Publications.

39. Food Standards Agency (2002) McCance and Widdowson's the Composition of Foods, 6th summary ed. Cambridge, UK: Royal Society of Chemistry.

40. Tan SP, Wenlock RW \& Buss DH (1985) Second Supplement to McCance and Widdowson's The Composition of Foods: Immigrant Foods. London: H.M. Stationery Office.

41. Hobhouse W (1994) The Classic 1000 Indian Recipes. London: Foulsham. 
42. DeMers J (2005) Authentic Recipes from Jamaica. Singapore: Periplus.

43. Schofield WN (1985) Predicting basal metabolic rate, new standards and review of previous work. Hum Nutr Clin Nutr 39, S41.

44. Goldberg GR, Black AE, Jebb SA, et al. (1991) Critical evaluation of energy intake data using fundamental principles of energy physiology: 1. Derivation of cut-off limits to identify under-recording. Eur J Clin Nutr 45, 569-581.

45. Black AE (2000) Critical evaluation of energy intake using the Goldberg cut-off for energy intake:basal metabolic rate. A practical guide to its calculation, use and limitations. Int J Obes Relat Metab Disord 24, 1119-1130.

46. Li L, Manor O \& Power C (2004) Are inequalities in height narrowing? Comparing effects of social class on height in two generations. Arch Dis Child 89, 1018-1023.

47. McKeigue PM, Marmot MG, Adelstein AM, et al. (1985) Diet and risk factors for coronary heart disease in Asians in northwest London. Lancet ii, 1086-1090.

48. Smith Z, Knight T, Sahota TP, et al. (1993) Dietary patterns in Asian and Caucasian men in Bradford: differences and implications for nutrition education. J Hum Nutr Diet 6, 323-333.

49. Field AE, Peterson KE, Gortmaker SL, et al. (1999) Reproducibility and validity of a food frequency questionnaire among fourth to seventh grade inner-city school children: implications of age and day-to day variation in dietary intake. Public Health Nutr 2, 293-300.

50. Gregory L \& Lowe S (2000) National Diet and Nutrition Survey: Young People Aged 4 to 18 Years. London: The Stationery Office.

51. Ortega RM, Requejo AM, Andres P, et al. (1995) Relationship between diet composition and body mass index in a group of Spanish adolescents. Br J Nutr 74, 765-773.

52. Garaulet M, Martinez A, Victoria F, et al. (2000) Difference in dietary intake and activity level between normal-weight and overweight or obese adolescents. J Pediatr Gastroenterol Nutr 30, 253-258.

53. Owen CG, Nightingale CM, Rudnicka AR, et al. (2009) Ethnic and gender differences in physical activity levels among 9-10-year-old children of white European, South Asian and African-Caribbean origin: the Child Heart Health Study in England (CHASE Study). Int J Epidemiol 38, 1082-1093.
54. Owen CG, Whincup PH, Orfei L, et al. (2009) Is body mass index before middle age related to coronary heart disease risk in later life? Evidence from observational studies. Int $J$ Obes (Lond) 33, 866-877.

55. Gupta R, Misra A, Pais P, et al. (2006) Correlation of regional cardiovascular disease mortality in India with lifestyle and nutritional factors. Int $J$ Cardiol 108, 291-300.

56. Willett W, Manson J \& Liu S (2002) Glycemic index, glycemic load, and risk of type 2 diabetes. Am J Clin Nutr 76, 274S-280S.

57. Enstrom JE, Kanim LE \& Klein MA (1992) Vitamin C intake and mortality among a sample of the United States population. Epidemiology 3, 194-202.

58. Harding AH, Wareham NJ, Bingham SA, et al. (2008) Plasma vitamin $\mathrm{C}$ level, fruit and vegetable consumption, and the risk of new-onset type 2 diabetes mellitus: the European Prospective Investigation of Cancer-Norfolk prospective study. Arch Intern Med 168, 1493-1499.

59. Pittas AG, Lau J, Hu FB, et al. (2007) The role of vitamin D and calcium in type 2 diabetes. A systematic review and meta-analysis. J Clin Endocrinol Metab 92, 2017-2029.

60. Luong KV \& Nguyen LT (2006) Vitamin D and cardiovascular disease. Curr Med Chem 13, 2443-2447.

61. Wang TJ, Pencina MJ, Booth SL, et al. (2008) Vitamin D deficiency and risk of cardiovascular disease. Circulation 117, 503-511.

62. Tai ES \& Tan CE (2005) Interaction between genetic and dietary factors affecting cardiovascular risk. Asia Pac J Clin Nutr 14, 72-77.

63. Choy PC, Siow YL, Mymin D, et al. (2004) Lipids and atherosclerosis. Biochem Cell Biol 82, 212-224.

64. Pereira MA \& Ludwig DS (2001) Dietary fiber and body-weight regulation. Observations and mechanisms. Pediatr Clin North Am 48, 969-980.

65. Yang EJ, Kerver JM, Park YK, et al. (2003) Carbohydrate intake and biomarkers of glycemic control among US adults: the third National Health and Nutrition Examination Survey (NHANES III). Am J Clin Nutr 77, 1426-1433.

66. Parillo M \& Riccardi G (2004) Diet composition and the risk of type 2 diabetes: epidemiological and clinical evidence. Br J Nutr 92, 7-19.

67. Liu S, Manson JE, Stampfer MJ, et al. (2000) Whole grain consumption and risk of ischemic stroke in women: a prospective study. JAMA 284, 1534-1540. 